\section{Patrick Eldin Bernard Cornillon Dominique Mornet Jean J. Léger}

\section{ADRESSE}

P. F.ldin : chercheur post-doctoral. B. Cornillon : chargé de recherche à l'Insern. D. Mornet: direcleur de recherche au (inrs. J.J. Léger: direcleur de recherche au C'ms, direcieur de l'C'. 300 Inserm. Inserm U. 300, Faculté de pharmacie, 15, avenue (Charles-Flahault, 34065 Montpellier Cedex, France.

\title{
Une nouvelle jeunesse pour les myosines
}

Des progrès majeurs ont permis le renouvellement des connaissances dans le domaine des "moteurs biologiques": les deux premières structures cristallines de la tête de la myosine viennent d'être publiées; de nouveaux tests de mobilité permettent de déterminer le mouvement et la force développée par une molécule de myosine unique le long du filament d'actine ; les progrès de l'ingénierie des protéines et des échanges entre sous-unités de myosine mettant à disposition des molécules chimères de myosine permettent l'étude de la fonction des chaînes lourdes et légères de la myosine et du rôle de certains domaines. La découverte de mutations ponctuelles dans la chaîne lourde de myosine ventriculaire de patients atteints de cardiomyopathie hypertrophique familiale donne une nouvelle dimension à ce domaine accessible maintenant à de nouvelles méthodes d'exploration.

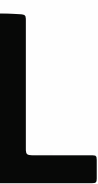

a contraction musculaire, phénomène fondamental au cours duquel l'énergie chimique (ATP) est transformée en énergie mécanique (mouvement), a fait l'objet de nombreuses études au cours des vingt dernières années. Deux protéines, la myosine et l'actine, jouent un rôle clé dans tout système contractile ou motile.

La structure tridimensionnelle de l'actine, molécule globulaire de $42 \mathrm{kDa}$, a été publiée il y a quatre ans $[1,2]$ et on a déduit une image précise de l'arrangement de l'actine en filaments. Les connaissances sur la myosine semblaient alors en retrait. On connaît pourtant depuis longtemps l'importance de cette molécule dont les parties globulaires, composées de plusieurs chaînes polypeptidiques (dites "têtes" ou sous-fragment 1 , de l'ordre de $120 \mathrm{kDa}$ ), constituent un des prototypes de moteurs biologiques. La grande taille de la molécule de myosine entière ( $500 \mathrm{kDa})$, sa structure hexamérique complexe (2 chaînes lourdes et 2 paires de chaînes légères), son existence en multiples isoformes et leur coexpression dans les différents types de muscles (squelettique, cardiaque et lisse) ont certainement constitué des barrières majeures aux études. L'hétérogénéité structurale et la plasticité conformationnelle de la molécule de myosine ont rendu tout particulièrement difficile la recherche de conditions efficaces de cristallisation. Au fil des années, de nombreuses isomyosines de différentes familles ont, certes, été de mieux en mieux caractérisées quant à leurs performances enzymatiques, leurs distributions respectives dans les différents types de muscles ou tissus considérés, leurs structures primaires en acides aminés et, enfin, leurs gènes respectifs [3, 4]. Cependant, encore très récemment, 


\section{RÉFÉRENCES}

1. Kabsch W, Mannherz HG, Suck D, Pai EF, Holmes KC. Atomic structure of the actin: DNase I complex. Nature 1990; 347 : 37-43.

2. Holmes KC, Popp D, Gebhard W, Kabsch. Atomic model of the actin filament. Nature $1990 ; 347: 448$.

3. McNally EM, Kraft R, Bravo-Zehnder $M$, Taylor DA, Leinwand LA. Full-length rat alpha and beta cardiac myosin heavy chain sequences: comparisons suggest a molecular basis for functional differences. $J \mathrm{Mol}$ Biol $1989 ; 210$ : 665-71.

4. Jaenicke $T$, Diederich $\mathrm{KW}$, Haas $\mathrm{W}$, Schleich J, Lichter P, Pfordt M, Bach A, Vosberg HP. The complete sequence of the human $\beta$-myosin heavy chain gene and a comparative analysis of its product. Genomics $1990 ; 8: 194-206$

5. Rayment I, Rypniewski WR, Schmidt-Bãse $\mathrm{K}$. Smith R, Tomchick DR, Benning MM, Winkelmann DA, Wesenberg G, Holden HM. Three dimensional structure of myosin subfragment-1: a molecular motor. Science $1993 ; 261: 50-8$

6. Rayment I, Holden HM, Whittaker M, Yohn CB, Lorenz M, Holmes KC, Milligan RA. Structure of the actin-myosin complex and its implications for muscle contraction. Science 1993 ; 261 : 58-65.

7. Xie X, Harrison DH, Schlichting I, Sweet $\mathrm{RM}$, Kalabokis VN, Szent-Györgyi AG, Cohen S. Structure of the regulatory domain of scallop myosin at $2.8 \mathrm{~A}$ resolury domain of scallop myosin at
tion. Nature $1994 ; 368: 306-12$.

8. Ishijima A, Doi T, Sakurada K, Yanagida T. Sub-piconewton force fluctuations of actomyosin in vitro. Nature 1991 ; 352: 301-6.

9. Finer JT, Simmons RM, Spudich JA. Single myosin molecule mechanics: piconewton forces and nanometer steps. Nature l'insuffisance des données structurales sur la myosine ainsi que sur les différences entre isomyosines a freiné l'étude des relations structure/fonction, faisant clairement obstacle à une réelle compréhension du phénomène contractile au plan moléculaire.

Des résultats importants viennent de donner une accélération subite aux connaissances sur les myosines dans quatre secteurs différents: (1) la structure tridimensionnelle de la tête globulaire de la myosine du muscle squelettique de poulet $[5,6]$ et celle du domaine régulateur de la myosine de coquille Saint-Jacques [7] ont été résolues à 2,8 $\AA$ par cristallographie aux rayons $\mathrm{X} ;(2)$ des tests de motilité in vitro ont permis la mesure intégrée et précise des capacités enzymatiques et motrices d'une molécule de myosine isolée $[8,9] ;(3)$ des données expérimentales directes ont été obtenues sur les rôles fonctionnels respectifs des chaînes lourdes et légères de myosine [10-14] ; (4) dans une atteinte congénitale grave du myocarde, la cardiomyopathie hypertrophique familiale $(\mathrm{CHF})$, on a mis en évidence des mutations ponctuelles de la myosine $\beta$, l'isoforme cardiaque de myosine exprimée dans le ventricule et dans le muscle squelettique lent $[15,16]$.

Nous appuyant sur l'exposé des résultats apportés dans ces quatre secteurs différents, nous nous efforcerons de souligner leurs interrelations mutuelles et leurs retombées actuelles et à venir sur la compréhension moléculaire du phénomène contractile. Nous discuterons enfin de l'impact fonctionnel des variations entre isomyosines et des mutations observées dans les myosines de certains cardiomyopathes.

\section{Structure tridimensionnelle de la tête de myosine}

La cristallisation de la tête de myosine squelettique de poulet a été obtenue après dix ans d'efforts par l'équipe de Ivan Rayment $[5,6]$. Cinquante kilos de "blanc" de poulet (près de 300 poulets) ont été nécessaires pour préparer $1 \mathrm{~kg}$ de myosine et isoler les quelques centaines de grammes de produit à cristalliser. La tête de myosine $(\mathrm{S} 1)$ a été préparée par l'action de la papaïne sur la myo- sine native. Sl est composé d'un long fragment de la chaîne lourde (les 843 résidus amino-terminaux sur les 1935 résidus constituant la chaîne entière), et des deux chaînes légères associées, les chaînes essentielles ELC (essential light chains) et les chaînes régulatrices RLC (regulatory light chains), respectivement de 25 et $17 \mathrm{kDa}$ (figure 1A). L'astuce qui a permis d'obtenir cette cristallisation du Sl a été la méthylation réductrice du Sl conduisant à la méthylation de près de $98 \%$ des chaînes latérales des lysines contenues dans la molécule native. Cette modification chimique du Sl assure une meilleure stabilité structurale et une protection contre la protéolyse endogène; ce traitement s'est avéré indispensable au succès de la cristallisation. L'analyse de diffraction des rayons $\mathrm{X}$ par le cristal de $\mathrm{S} 1$ a permis la résolution de sa structure à $2,8 \AA$. De la confrontation des modèles structuraux du $\mathrm{Sl}$ et de l'actine, et de l'image de reconstitution de l'actine décorée par le S1, est sortie une structure prédictive de l'interface actine/myosine. La tête de la myosine ou Sl est composée de deux parties: une partie distale plutôt globulaire ne contient que de la chaîne lourde; l'autre partie, plutôt filiforme, contient les deux chaînes légères fixées à la chaîne lourde (figure 1A). La partie globulaire distale de la tête de myosine ou $\mathrm{Sl}$ (165 $\AA$ en longueur, $65 \AA$ en largeur et $\sim 40 \AA$ en épaisseur) (partie supérieure de la structure sur la figure $1 A)$, est centrée autour d'un grand feuillet $\beta$ à sept brins (figure 1B), imbriquant les uns par rapport aux autres les éléments structuraux issus des trois parties contiguës constituant l'ensemble du Sl. Ces trois parties, de masses moléculaires respectives 23,50 et $20 \mathrm{kDa}$, correspondent, en fait, aux fragments de la chaîne lourde produits par l'action ménagée de la trypsine. La présence de sites flexibles, exposés sur la chaîne lourde de myosine, tout comme leur implication dans les interactions avec l'actine et l'hydrolyse de l'ATP, est connue depuis longtemps [17]. I.a forte mobilité de ces boucles a empêché la détermination de leur structure cristalline. La partie globulaire distale de la tête de la myosine contient, en fait, le site de fixation du nucléotide et les zones d'interaction avec 


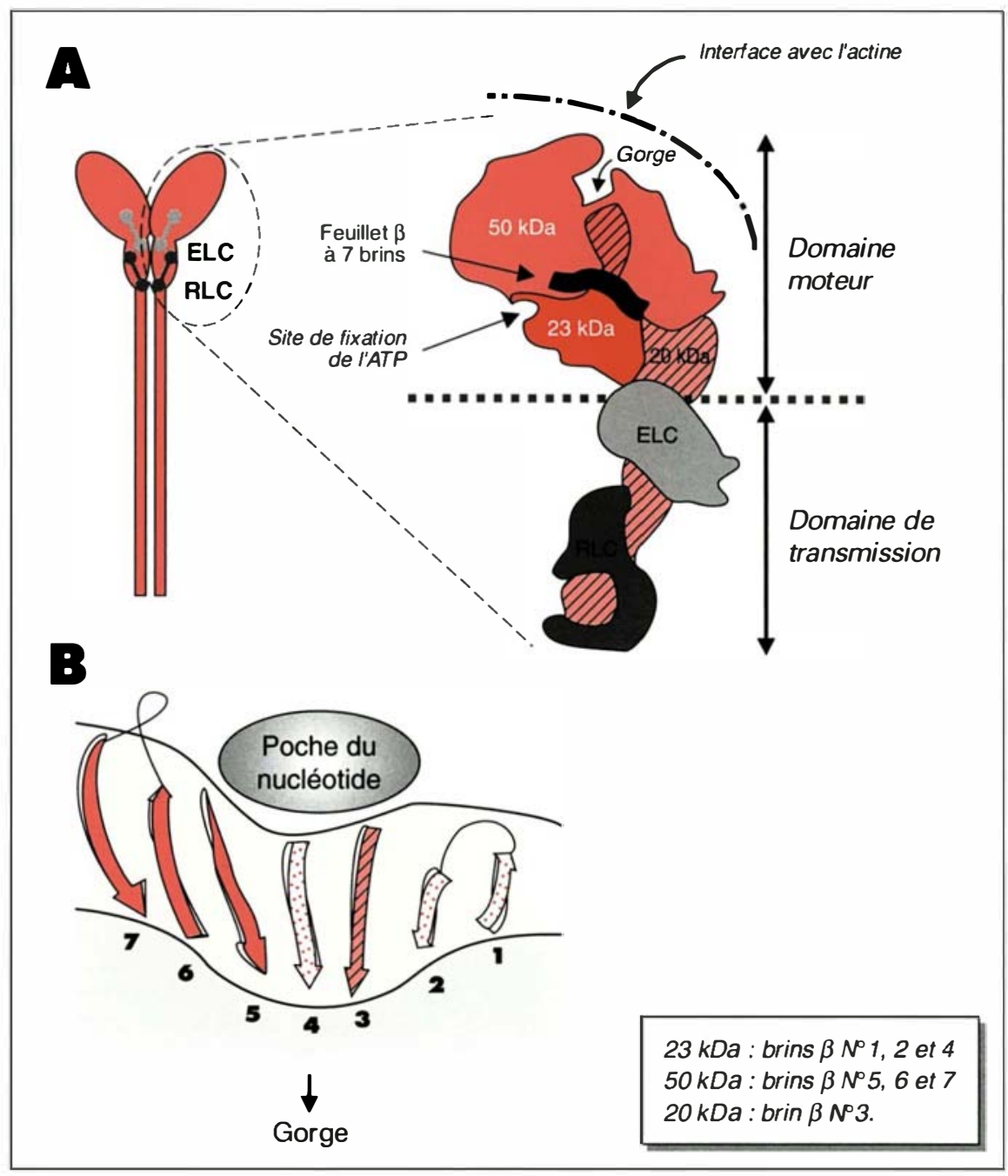

l'actine. Il s'agit donc du "domaine moteur ", partie active de la molécule de myosine. L'autre domaine de la tête révélé par le cristal est un ensemble structural oblong, constitué par l'enroulement des chaînes légères essentielles et régulatrices autour d'une longue hélice de $85 \AA$, correspondant à la partie C-terminale du fragment protéolytique $20 \mathrm{kDa}$ de la chaîne lourde de myosine (partie inférieure de la structure sur la figure 1A). Ce domaine, allongé et courbe, est susceptible de participer à la régulation de l'activité enzymatique et à la transmission de la force motrice produite par le domaine moteur au reste de la molécule; il constitue ce qu'on peut appeller le "domaine de transmission" de la myosine.
Des caractéristiques importantes des zones fonctionnelles de la tête de myosine émergent de cette structure tridimensionnelle: (1) le motif structural central est constitué par des brins $\beta$ issus des trois fragments protéolytiques de la chaîne lourde (23, 50 et $20 \mathrm{kDa}$ ); ce feuillet $\beta$ à sept brins (figure 1B) est le cœur ou le pivot central de la molécule de myosine ; (2) les jonctions entre les segments de 23 et $50 \mathrm{kDa}$ (en relation avec la poche de fixation du nucléotide) et entre les segments de 50 et $20 \mathrm{kDa}$ (impliqués dans l'interface avec l'actine) sont situées sur des faces opposées de la molécule, de chaque côté du feuillet à sept brins. Les phosphates de l'ATP s'associent à une structure $P$-loop commune aux adénylyl kinases et aux protéines Ras ; (3) le
Figure 1. A. Schéma déduit de la structure tridimensionnelle de la tête de myosine de muscle squelettique de poulet. La tête de myosine est composée de deux domaines contigus: le domaine moteur et le domaine de transmission $[5,6]$. Les trois fragments protéolytiques de la chaîne lourde $(23,50$, et $20 \mathrm{kDa} d u$ résidu $N$-terminal au résidu $C$-terminal) ont été colorés différemment. Les chaînes légères essentielles (ELC) et régulatrices (RLC) ont également été distinguées. Les sites fonctionnels principaux ont été indiqués. $B$. Détails de la poche centrale (feuillet) du domaine moteur, constitué de sept brins $\beta$ issus des diverses parties de la chaîne lourde. On suppose que le nucléotide se fixe dans cette poche. fragment $50 \mathrm{kDa}$ est scindé en deux lobes par une profonde crevasse ou gorge; les parties exposées de chacun des lobes du fragment 50 kDa participent à l'interaction avec l'actine.

Les prédictions concernant la structure de l'interface actine/myosine montrent que la meilleure adéquation entre les structures de l'actine et de la myosine est obtenue en imposant la fermeture et l'ouverture de la gorge dans le fragment $50 \mathrm{kDa}$. Cette gorge s'étend du plancher de la poche du nucléotide à l'interface avec l'actine. Un tel arrangement suggère que des changements de conformation induits par le nucléotide pourraient être à la base de la modulation de l'affinité de la myosine pour l'actine, à travers le feuillet central à sept brins. L'aspect com- 


\section{RÉFÉRENCES}

10. Lowey S, Waller GS, Trybus KM. Func tion of skeletal muscle myosin heavy and light chain isoforms by an in vitro motility assay. $J$ Biol Chem 1993; 268 : 20414-8.

11. Lowey S, Waller GS, Trybus KM. Skeletal muscle myosin light chains are essential for physiological speeds of shortening. Nature 1993; 365: 454-6.

12. Itakura $\mathrm{S}$, Yamakawa $\mathrm{H}$, Toyoshima $\mathrm{YY}$ Ishijima A, Kojima T, Harada $Y$, Yanagida T, Wakabayashi T, Sutoh K. Force generating domain of myosin motor. Biochem Biophys Res Commun 1993; 196: 1504-10.

13. Uyeda TQP, Spudich JA. A functional recombinant myosin II lacking a regulatory light chain binding site. Science 1993; 262: 1867.70 .

14. Uyeda TQP, Ruppel KM, Spudich JA. Enzymatic activities correlate with chimaeric substitutions at the actin-binding face of myosin. Nature 1994; 368: 567-9.

15. Tanigawa GJ, Kass SS, Vosberg HP, Seidman JG, Seidman CE. A molecular basis for familiar hypertrophic cardiomyopathy: an $\alpha / \beta$ cardiac myosin heavy chain hybrid gene. Cell 1990 ; 62 : $991-8$.

16. Geisterfer-Lowrance AA, Kass S, Tanigawa G, Vosberg HP, McKenna W, Seidman CE, Seidman JG. A molecular basis for familial hypertrophic cardiomyopathy: a $\beta$ cardiac myosin heavy chain gene missense mutation. Cell 1990; 62: 999-1006.

17. Audemard E, Bertrand R, Bonet A Chaussepied P, Mornet D. Pathway for the communication between the ATPase and actin sites in myosin. I Muscle Res Cell Motil $1988 ; 9: 197-218$

18. Trayer IP, Trayer HR, Levine BA. Evidence that the N-terminal region of A1-light chain of myosin interacts directly with the C-terminal region of actin. Eur I Biochem pact de la partie N-terminale de la myosine, avec son enchevêtrement relatif de portions éloignées de la chaîne lourde en un dispositif central à sept brins, permet maintenant de comprendre pourquoi il était impossible de séparer les trois fragments de la tête sans les dénaturer et pourquoi cela entraînait la perte de la fonction enzymatique. La structure cristalline disponible permet d'identifier l'importance fonctionnelle de certains résidus ou boucles d'acides aminés dans la tête de la myosine. Elle permet en particulier de déduire des informations sur les éventuelles conséquences fonctionnelles des diverses mutations observées dans la chaîne lourde de myosine ventriculaire des cardiomyopathes (voir figure 5). La structure obtenue a cependant quelques limites qu'il convient de souligner. Par exemple, la plupart des pontages chimiques réalisés dans la molécule de myosine native indiquent, curieusement, des distances entre certains acides aminés, dont la plupart ne sont pas compatibles avec les positions spatiales de ces mêmes résidus, déduites de la structure cristalline. La figure 2 résume quelquesunes de ces différences de distance entre l'un ou l'autre des groupes thiols (SH1 et $\mathrm{SH} 2$ ) réactifs du fragment $20 \mathrm{kDa}$ et des résidus des autres parties de la tête de myosine. Ces pontages ne sont possibles que si la molécule native est dans d'autres états de conformation que celui présenté par la structure cristallisée. Autrement dit, il faut admettre que la structure publiée ne représente qu'un des multiples états conformationnels de la tête de la myosine rencontrés séquentiellement au cours de la contraction. Il est probable, en outre, que la méthylation intense des lysines provoque une dilatation du volume moléculaire de la tête de myosine, l'éloignant de sa compacité en solution. Il faut également noter que d'autres parties de la chaîne lourde de la myosine, ou même la partie N-terminale de la chaîne légère ELC, modulent en solution l'interaction avec l'actine à différents stades du cycle contractile [18]. Des expériences récentes faites au laboratoire montrent, en effet, que des fragments contigus de la tête de myosine contenant les boucles sensibles à la protéolyse sont capables de se lier indépendamment à l'actine [19]. Des conformations particulières de la tête de la myosine doivent donc être envisagées pour permettre une telle interaction entre l'actine et la chaîne légère. Aussi la structure actuellement disponible dans laquelle la crevasse représente le site principal d'interaction avec l'actine ne rendelle compte que d'une des principales zones d'interaction entre l'actine et la myosine.

\section{Des tests de motilité in vitro}

En 1983, Sheetz et Spudich [20] ont montré qu'il était possible de suivre in vitro, par microscopie optique, le mouvement de filaments fluorescents d'actine (la crémaillère) catalysé par un petit groupe de molécules de myosine (le moteur) immobilisé sur un support solide et consommant de l'ATP (le carburant) (figure 3A). C.ela a permis de déterminer des vitesses relatives de déplacement de filaments d'actine mûs par un ensemble de molécules de myosine orientées de manière plus ou moins capricieuse. Les vitesses moyennes obtenues sont de l'ordre de quelques $\mu \mathrm{m} / \mathrm{s}$; elles sont caractéristiques d'un type donné de myosine et correspondent aux vitesses maximales de glissement entre les filaments d'actine et de myosine, mesurées dans le muscle entier. Ces expériences de motilité, effectuées avec les têtes de myosine uniquement, ont démontré que les têtes seules sont suffisantes pour engendrer une tension.

En 1988, Kishino et Yanagida [21] ont réussi à accrocher un filament d'actine à une fibre de verre très mince et très flexible afin de mesurer la force exercée sur cette fibre par des molécules de myosine placées à proximité. La mesure par effet piézoélectrique de la courbure de la fibre de verre permet de déterminer la force globale exercée par quelques molécules de myosine en présence d'ATP (figure 3B). Il en a été déduit que la force d'interaction élémentaire entre le filament d'actine et une molécule unique de myosine était de l'ordre de quelques piconewtons. Le progrès le plus récent, qui permet aujourd'hui d'étudier le fonctionnement des moteurs biologiques à 


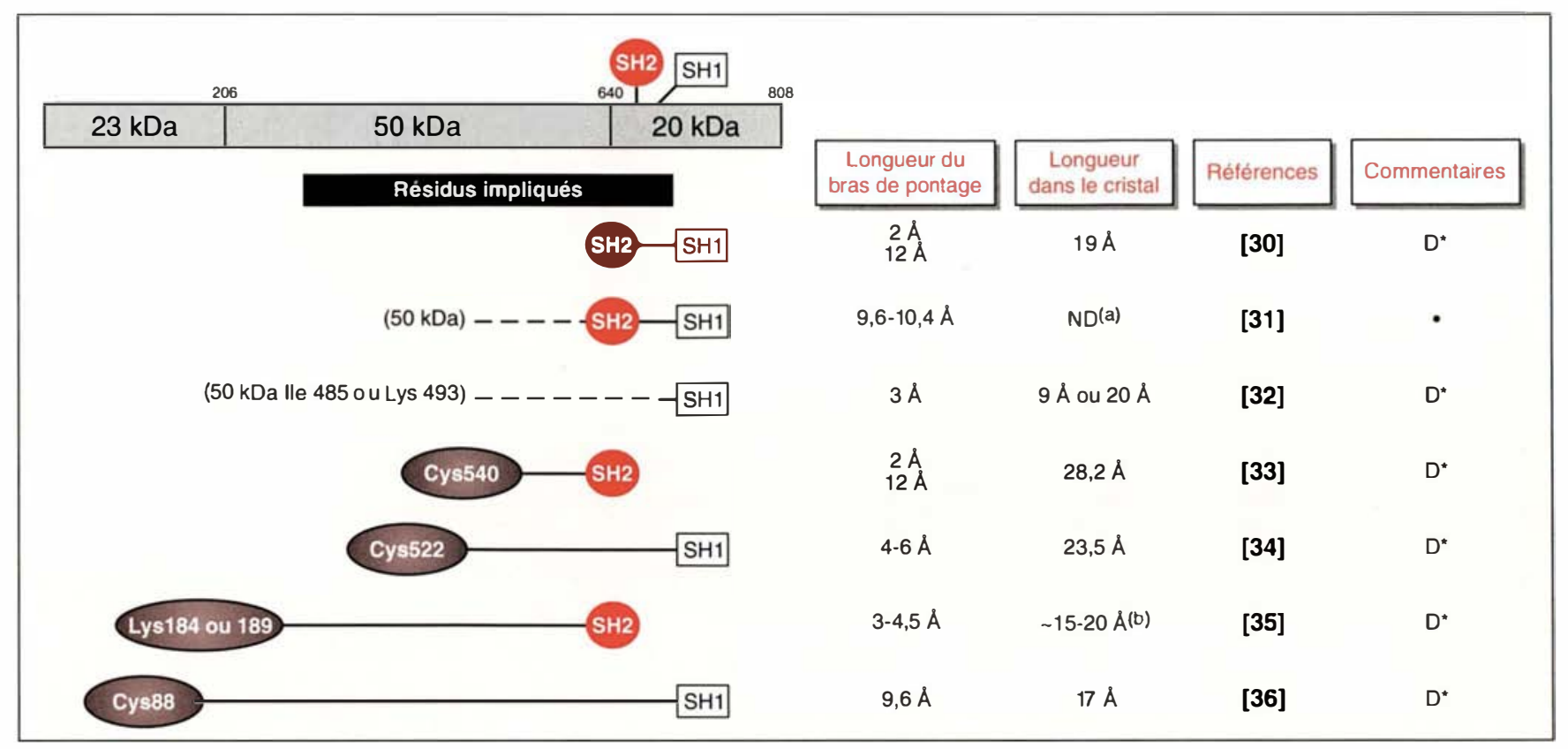

Figure 2. Liste de quelques réactions de pontage entre des groupes thiols réactifs de la chaîne lourde et d'autres résidus de la partie $\mathbf{N}$-terminale de la même chaîne. Des différences importantes de distances entre résidus mesurées par les pontages et ceux déduits de la structure cristalline (communication personnelle de l. Rayment) sont observées. (a) non déterminé; (b): distances déterminées graphiquement; $D^{*}$ : difficile à ponter sans réarrangement conformationnel.

l'échelon de molécules individuelles, résulte de l'emploi de "pinces optiques" [9]. Celles-ci sont des faisceaux lasers intenses qui piègent microscopiquement des billes de silice sur lesquelles ont été placés, soit le filament d'actine, soit des molécules de myosine (figure 3C). Ces dispositifs permettent de faire varier la charge exercée sur une seule molécule de myosine et de mesurer à la fois la vitesse et la force engendrées par le déplacement de cette myosine par rapport au filament d'actine. Ces dispositifs électroniques sophistiqués domnent un support expérimental direct à la théorie du glissement relatif des filaments d'actine et de myosine proposée dès 1954 (pour une revue, voir [22]). L'amplitude moyenne des déplacements observés $(11 \mathrm{~nm})$, ainsi que la valeur moyenne de la force produite $(3,4 \mathrm{pN})$ par une molécule de myosine de muscle squelettique rapide de poulet, confirment globalement le modèle de fonctionnement en quatre temps des moteurs moléculaires: (1) dissociation de la myosine du filament d'actine à la suite de la fixation de l'ATP (premier temps); (2) hydrolyse de l'ATP en ADP et Pi et fixation de la myosine au filament d'actine (deuxième temps); (3) libération du phosphate et rotation de la tête de la myosine entraînant le déplacement du filament d'actine (troisième temps); (4) production de la force et libération de l'ADP (quatrième temps). Le processus de transfert d'énergie chimique en énergie mécanique effectué par les molécules de myosine a un rendement de l'ordre de $40 \%$.

Le déplacement moyen observé à partir des études de motilité de la tête de la myosine $(11 \mathrm{~nm})$ est le double de celui déduit du cristal de Rayment [5, 6]. Dans ce dernier modèle, l'ouverture de la crevasse après fixation de l'ATP et relargage de l'actine ne permet, en effet, qu'un déplacement de la myosine de l'ordre de $5 \mathrm{~nm}$, suggérant à nouveau que la structure déterminée par les rayons $X$ ne représente qu'un des nombreux états de transition confor- mationnelle auxquels est soumise la tête de myosine au cours du cycle contractile. Les mesures les plus récentes de motilité indiquent clairement que la force momentanée produite a, en fait, une durée plus longue que le déplacement de la tête de myosine et que l'augmentation de la charge appliquée à une molécule de myosine réduit sa vitesse de déplacement. Cette utilisation progressive de l'énergie chimique résulte probablement d'un certain découplage entre la formation des ponts actinemyosine et l'hydrolyse de l'ATP. Ce découplage est sans doute à l'origine de la grande variabilité observée dans les déplacements (7 à $17 \mathrm{~nm}$ ) et dans les forces (1 à $7 \mathrm{pN}$ ) mesurés pour des molécules individuelles de myosine. Naturellement, il faut prendre en compte d'autres facteurs comme les perturbations résultant du mouvement brownien, les contraintes mécaniques induites par la fixation des têtes de myosine et le rôle propre du filament d'actine. Il faut également considérer les limites expérimentales 


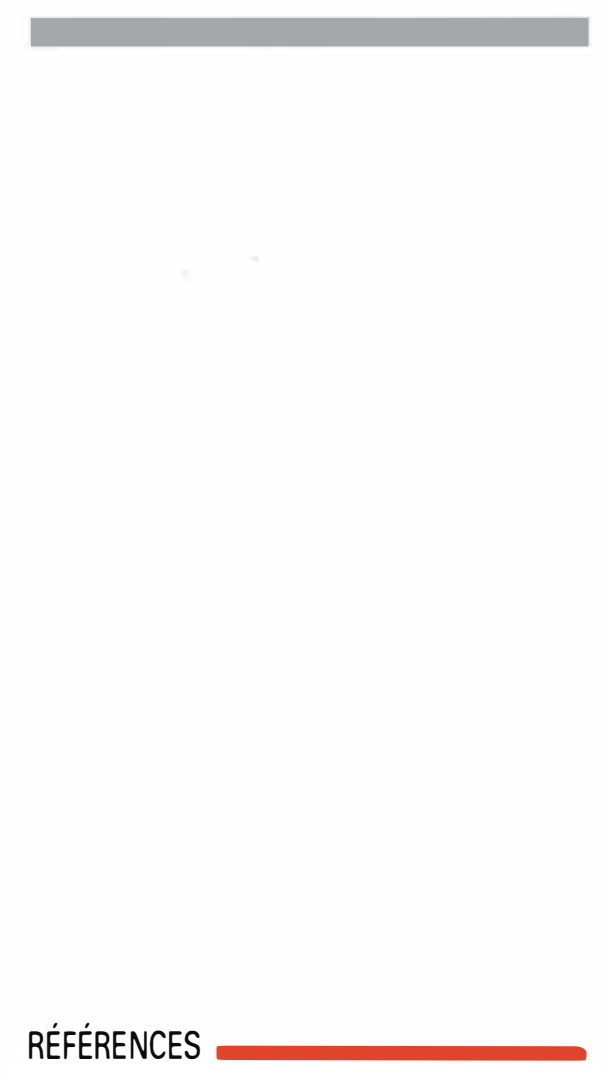

19. Eldin P, Le Cunff M, Vosberg HP, Mornet D, Léger JJ. Mapping of the actomyosin interfaces. Proc Nall Acad Sci USA 1994; 91 : 2772-6.

20. Sheetz MP, Spudich JA. Movement of myosin-coated fluorescent beads on actin cables in vitro. Nature $1983 ; 303: 31-5$.

21. Kishino A, Yanagida T. Force measurements by micromanipulation of a single actin filament by glass needles. Nature 1988; 334: $74-6$

22. Huxley HE. Sliding filaments and molecular motile systems. $\int$ Biol Chem 1990; 265 . 8347-50.

23. Harrington WF, Ueno $H$. Structural transitions in myosin and the origin of contractile force in muscle. Biopolymers 1987; 26: 81-98.

24. Choi-Man Ng, Ludescher RD. Microsecond rotational dynamics of F-actin in actoS1 filaments during ATP hydrolysis. Biochemisity 1994; 33: 9098-104.

25. Trybus KM, Waller GS, Chatman TA Coupling of ATPase activity and motility in smooth muscle myosin is mediated by the regulatory light chain.J Cell Biol 1994; 124 : 963-9.

26. Hengstenberg C, Schwartz K. Molecular genetics of familial hypertrophic cardiomyopathy. J Mol Cell Cardiol 1994; 26: 3-10.

27. Vybiral T, Deitiker PR, Roberts $R$ Epstein HF. Accumulation and assembly of myosin in hypertrophic cardiomyopathy with the 403-Arg to Gin beta myosin heavy

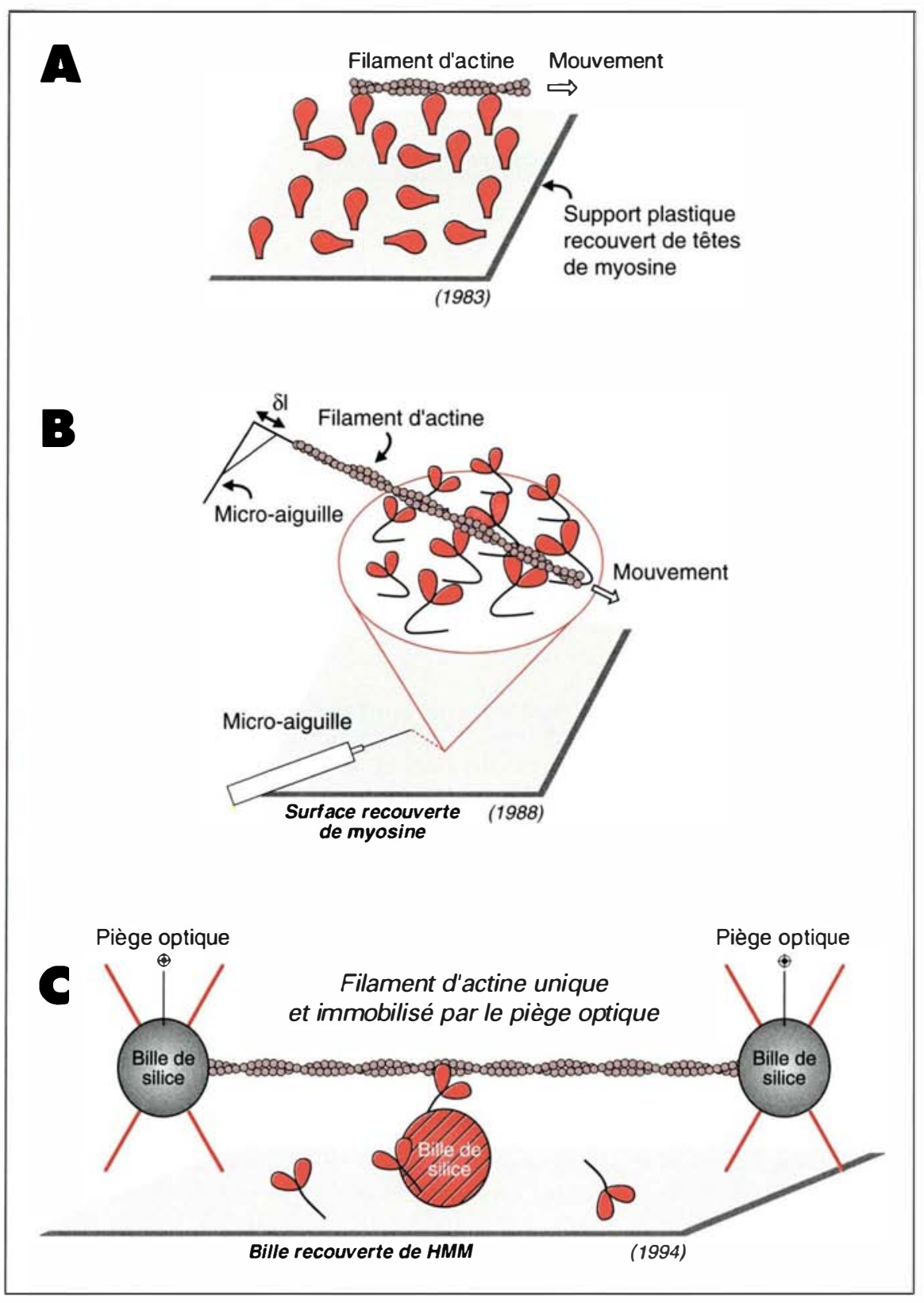

Figure 3. Techniques permettant d'observer le mouvement des molécules de myosine par microscopie optique et de mesurer la force mécanique produite. A. Les molécules de myosine sont fixées sur un support plastique transparent. Le mouvement relatif de filaments fluorescents $d^{\prime}$ actine est suivi après ajout d'ATP, soit directement, soit par enregistrement sur vidéocaméra (voir [9]). B. Les molécules de myosine sont toujours fixées sur un support solide. Les filaments $d^{\prime}$ actine sont piégés à une fibre de verre très fine, dont le déplacement ( $\delta 1)$ sera mesuré lors de l'interaction entre le filament d'actine et les myosines, après ajout d'ATP [21]. C. Les molécules de myosine et les extrémités des filaments d'actine sont accrochées à des billes de silice. Ces billes sont piégées (bloquables ou déplaçables) par des faisceaux lasers indépendants. Une fois atteint le positionnement d'une seule molécule de myosine auprès d'un filament unique d'actine, de l'ATP est ajouté. Le déplacement de la tête de myosine piégée et la force exercée sur le filament d'actine peuvent être mesurés par l'intermédiaire des faisceaux lasers ! II s'agit là de l'étude des capacités motrices d'une molécule unique de myosine [9]. HMM: heavy meromyosin. 
des méthodes utilisées. Des molécules de myosine mal orientées sur la bille de silice peuvent, par exemple, hydrolyser "pour rien" l'ATP si elles sont trop éloignées du filament d'actine. Dans le muscle, de telles variations peuvent exister dans l' "état» de contraction des molécules individuelles, dans leur géométrie réciproque, dans leurs interactions résiduelles. Dans la limite de leur résolution, les méthodes les plus récentes indiquent que le moteur actomyosine produit une force constante durant le cycle d'hydrolyse de l'ATP. Ce couplage mécanochimique "mou" ne peut être atteint que si la formation d'un pont actinemyosine peut induire non pas une impulsion unique, mais une suite d'interactions génératrices de force pour un seul cycle d'hydrolyse de l'ATP. Le découplage, qui existe probablement ainsi entre le temps moteur (hydrolyse de l'ATP) et le déplacement induit (déplacement du filament d'actine), laisse supposer que des changements conformationnels de la tête de la myosine se produisent successivement tout le long du cycle. Une réaction élastique (type force de rappel par raccourcissement) de la partie S2 de la myosine, qui joint la tête de la myosine à la partie C-terminale associée dans le filament épais de myosine, peut également être à l'origine de ce maintien prolongé de l'impulsion initiale. Cette hypothèse de deux composants, l'un actif et l'autre passif, dans le mécanisme de la contraction a été formulée, il y a plusieurs années [23]. Enfin, il est clair que le filament d'actine n'est pas un partenaire inerte. Des fluctuations conformationnelles actives de grande ampleur et de longue durée se produisent lors de la contraction [24].

\section{Le fonctionnement des chaînes lourdes et légères, celui des isomyosines}

Des progrès ont été réalisés très récemment sur la compréhension du fonctionnement relatif des chaînes légères et lourdes de myosine, ainsi que sur la fonctionnalité de segments définis de chaînes lourdes. Ceux-ci ont été réalisés, notamment grâce à un renouvellement des techniques de dissociation et d'échange des chaînes légères dans les molécules de myosine de muscle squelettique, et grâce à l'étude comparative des diverses isoformes de myosine dont le type de chaînes lourdes est réglé au cours du développement. Le développement des méthodes d'expression de myosines non musculaires sous forme de molécules à composition chimérique de chaînes lourdes et légères et/ou de fragments hybrides de chaînes lourdes a permis d'effectuer également de nombreuses études de relations structurefonction des myosines non musculaires. Les mesures des activités enzymatiques et la détermination de la motilité de toutes ces myosines naturelles ou chimères donnent des indications sur le rôle des diverses sous-unités composant la molécule de myosine ou sur certains fragments de chaînes.

La figure 4 résume les expériences sur le rôle des chaînes légères dans la myosine de muscle squelettique de poulet [10]. L'élimination des chaînes légères ELC et RI.C diminue par un facteur 10 la vitesse de motilité des molécules de myosine "dénudées" dans le domaine de transmission de la tête de la myosine (figure 1A). Ces molécules sont capables d'hydrolyser relativement rapidement l'ATP en présence d'actine. L'ajout de la chaîne légère ELC ou de la chaîne légère RLC ou des deux (voir schémas de gauche à droite dans la figure 4) augmente significativement les capacités motrices des myosines reconstituées, leurs activités d'hydrolyse de l'ATP restant toujours proches des valeurs normales. Ces expériences indiquent clairement que les chaînes légères jouent un rôle direct dans le mécanisme de motilité et non dans le mécanisme d'hydrolyse de l'ATP proprement dit. L'analyse fine de la structure tridimensionnelle de la tête de la myosine suggère que les chaînes légères, fixées sur le manchon constitué par la partie C-terminale du segment 20 kI)a de la chaîne lourde, pourraient assurer la rigidité du domaine de transmission dans la tête de myosine. Une telle rigidité est sans doute nécessaire à la transmission efficace de la force produite dans la partie globulaire de la tête vers la partie fibrillaire de la myosine, là où les chaînes lourdes sont associées en filaments épais. Dans les myosines musculaires, les chaînes légères jouent donc un rôle essentiel dans la motilité, mais peu ou pas dans les activités enzymatiques qui seraient déterminées par les chaînes lourdes. D'autres expériences de même type (échange de chaînes lourdes et légères) ont également permis d'établir que les différentes isoformes environnementales des chaînes légères et lourdes sont suffisantes pour induire le continuum des vitesses de contraction des muscles et leur adaptation aux changements auxquels ils sont soumis lors de la myogenèse ou au cours de l'exercice musculaire (plasticité) [11].

Les résultats publiés très récemment sur le rôle joué par les chaînes légères dans les myosines non musculaires (la myosine du nématode Dictyostelium [14]) ou d'autres types de myosine musculaire (la myosine de coquille Saint-Jacques [7]) ou dans des myosines de muscle lisse [25] amènent à des conclusions apparemment différentes de celles obtenues avec les myosines de muscle squelettique de mammifères. Par exemple, l'élimination de chaînes légères, par empêchement de leur fixation à la suite de la délétion de la zone de chaîne lourde correspondante, ne modifie pas la motilité mais induit des changements majeurs dans les activités enzymatiques de la myosine de Dictyostelium (schéma central de la figure 4). Dans ces myosines, comme dans de nombreuses myosines non musculaires ou lisses, la régulation du calcium lors de la contraction est relayée par les chaînes légères et intervient directement dans l'hydrolyse de l'ATP en présence d'actine par un mécanisme de phosphorylation des chaînes légères. Ce système de régulation par le calcium est naturellement très différent de celui des muscles striés dont la régulation est effectuée principalement au niveau du filament fin par le système tropomyosine-troponine. La délétion sélective par génic génétique de la zone de la chaîne lourde de myosine de Dirtyostelium, fixant la chaîne légère RLC, montre que la régulation par la phosporylation de l'activité Mg-ATPasique dépendant de l'actine et de la motilité est relayée par la chaîne légère ELC (schéma de droite dans 


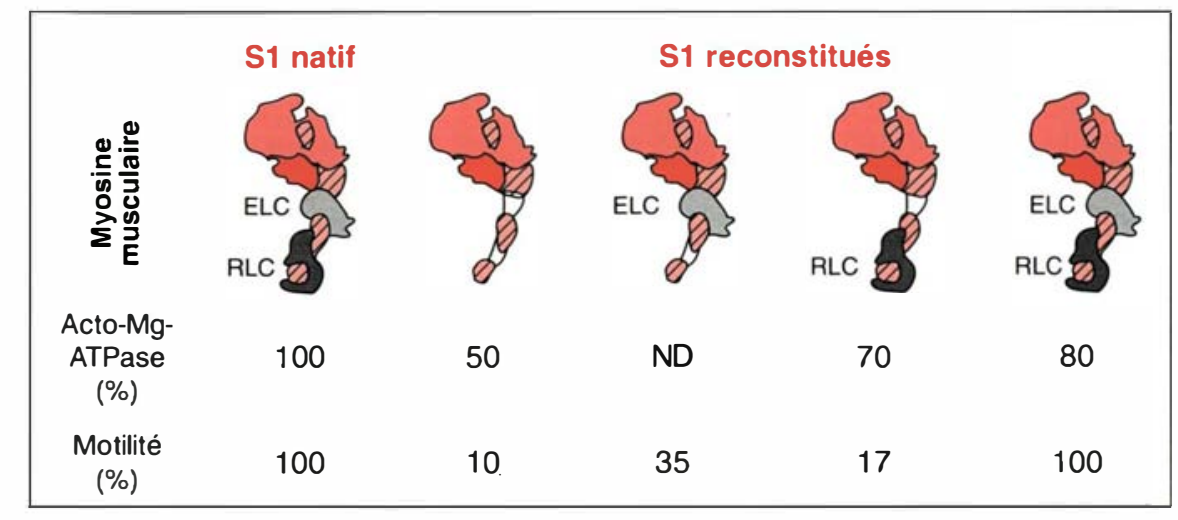

Figure 4. Expérience d'élimination et de reconstitution de chaînes légères dans la tête de la myosine de muscle squelettique rapide de poulet. Les chaînes légères essentielles (ELC) et régulatrices (RLC) peuvent être séparées des chaînes lourdes de la tête de myosine (sous-fragment S1) sous l'effet $d^{\prime}$ agents chaotropes. On peut ensuite ajouter les chaînes légères l'une après l'autre et reconstituer ainsi partiellement la structure native de la tête de myosine. On a mesuré les effets de l'élimination et de l'ajout des chaînes légères sur les activités ATPasiques dépendantes du magnésium en présence d'actine et sur les valeurs de la motilité des "S1 reconstitués" [10]. Des réductions marquées des valeurs de motilité par rapport aux molécules natives sont observées. Les deux types de chaînes légères jouent un rôle décisif dans la transmission de la force produite dans le domaine moteur de la myosine par I'hydrolyse de l'ATP. Les chaînes légères contribueraient à stabiliser le domaine de transmission et permettraient ainsi des transmissions efficaces entre le domaine moteur et la partie fibrillaire de la myosine (figure 1A). mutations in familial hypertrophic cardiomyopathy. Proc Nall Acad Si USA 1994; 91 : $589-93$

29. Sweeney HL, Straceski AJ, Leinwand LA, Tikunov BA, Faust L. Heterologous expression of a cardiomyopathic myosin that is defective in its actin interaction. J Biol Chem $1994 ; 269: 1603-5$.

30. Wells JA, Yount R. Chemical modification of myosin by active site trapping of metal-nucleotide with cross-linking reagent. Meth Enzymol 1982; 85: 93-113.

31. Rajasekharan KN, Sivaramakhrishnan $\mathrm{M}$, Burke M. Proximity and ligand induced movement of inter domain residues in myosin subfragment-1 containing trapped MgATP and MgPPi probed by multifunctional crosslinking. $J$ Biol Chem 1987; 262: 11207 14.

32. Muno D, Sekine T. Photocross-linking from dinitrophenylated $\mathrm{SH} 1$ in myosin head. Cross-linked site on $50 \mathrm{kDa}$ fragment. J Biochem 1988; 104: 427-32.

33. Chaussepied D, Morales MF, Kassab R. The myosin SH2-50 kilodalton fragment cross-link: location and consequences. Biochemisiny 1988; 27 : 1778-85.

34. Ue K. Intramolecular cross-linking of myosin subfragment-1 with bimane. Biochemisiry 1986; 26: 1889-94.

35. Sutoh K, Hiratsuka T. Spatial proximity of the glycine-rich loop and the SH2 thiol in myosin subfragment-1. Biochemistry 1988; 27: 29649

36. Lu RC, Wong A. Glutamic acid-88 is close to $\mathrm{SHl}$ in the tertiary structure of myosin subfragment-1. Biochemistry 1989; 28 : 48269 .

la figure 5). Un rôle inhibiteur de l'activité enzymatique pour la chaîne légère RLC et le segment de chaîne lourde correspondant ainsi qu'un rôle structural pour le mouvement de la myosine non musculaire le long de l'actine en ont été déduits [13]. La parution en avril 1994 de la structure tridimensionnelle du domaine régulateur de la myosine de coquille Saint-Jacques [7] donne des indications nouvelles sur cette zone de la myosine, caractéristique de ce type particulier de myosines musculaires. Le cristal, formé des deux chaînes légères ELC et RLC et du fragment correspondant de chaîne lourde, indique que le site de fixation du calcium (main de type EF) est essentiellement composé de résidus d'acides aminés appartenant à ELC. La stabilisation de la fixation de l'ion n'est obtenue que par des résidus provenant de l'autre chaîne légère et de la chaîne lourde. Ce complexe ternaire subtil fixant le calcium (dont la structure est analogue à la partie oblongue du S1 musculaire) est stabilisé par une interaction entre la partie C-terminale d'ELC et la partie $\mathrm{N}$ - terminale de RLC. On comprend mieux pourquoi cet ensemble compact doit être entier pour assurer sa fonction complète!

Les résultats concernant l'expression de la tête tronquée de myosine de Diclyostelium (schéma central de la figure 5 [12]) confirment les données structurales de Rayment el al. [5, 6]. Le domaine moteur (figure 1A) correspond bien à la partie distale globulaire de la tête de myosine: il est suffisant pour hydrolyser l'ATP et déplacer le filament d'actine. Des expériences encore plus récentes, échangeant par génie génétique un segment court de la chaîne lourde jouxtant les fragments 50 et $20 \mathrm{kDa}$ de diverses têtes de myosine [14], indiquent que ce segment flexible des chaînes lourdes est clairement l'un des déterminants des valeurs des activités Mg-ATPasiques dépendantes de l'actine (figure 6). Cette zone des chainnes lourdes est une des sept zones de divergence primaire observées entre les diverses chaînes lourdes de myosine [3]; malgré son caractère fonctionnel, sa longueur et sa séquence primaire sont extrême- 


\begin{tabular}{ccccc|}
$\begin{array}{c}\text { Myosine } \\
\text { non-musculaire }\end{array}$ \\
$\begin{array}{c}\text { Acto-Mg-ATPase } \\
\text { (\%) } \\
\text { Motilité (\%) }\end{array}$ & 100 & 10000 & $760 \quad 250$ \\
$(1-758)$ & 90 & 50 \\
\hline
\end{tabular}

Figure 5. Construction de myosines chimères (systèmes non musculaires). Les chaines lourdes de myosine de muscle squelettique ou de Dictyostelium peuvent être tronquées ou supprimées par génie génétique. Les fragments obtenus sont ensuite exprimés dans Dictyostelium où ils sont éventuellement capables de se réassocier à des chaînes légères endogènes. La troncature de la partie $C$-terminale du S1 (schéma au centre de la figure, [12]) ou l'élimination sur la chaîne lourde de la zone de liaison de la chaîne légère régulatoire RLC (schéma sur la partie droite de la figure, [13]) induisent des augmentations importantes des activités ATPasiques dépendantes du magnésium en présence d'actine et des variations plus modérées des valeurs de la motilité, comparées aux molécules natives. Cela suggère que, dans les myosines non musculaires, les chaînes légères et la partie C-terminale de la chaîne lourde (domaine de transmission) interviendraient directement dans la régulation de l'hydrolyse de I'ATP qui débute dans le domaine moteur. L'effet observé dans le cas de la délétion est modulé par l'action de la kinase, même en l'absence de la chaîne légère RLC. La structure cristalline fine du complexe chaînes légères-chaînes lourdes des myosines non musculaires [7] permet de comprendre comment le complexe ternaire (chaînes lourdeschaînes légères) peut intervenir dans la régulation de l'hydrolyse de l'ATP.

ment variables d'un type de myosine à un autre. Ces résultats permettent de commencer à comprendre comment de faibles variations de séquences primaires entre isoformes (plus de $95 \%$ de conservation) peuvent entraîner des différences fonctionnelles subtiles entre les isomyosines lors du développement ou lors de certains développements physiopathologiques.

\section{Mutations de la myosine $\beta$ et cardiomyopathie hypertrophique familiale}

Les observations faites par le groupe de Boston sur la présence de mutations ponctuelles dans les gènes codant pour les chaînes lourdes de myosine ventriculaire de patients atteints de cardiomyopathie hypertrophique familiale (CHF) ont été à l'origine de toute une série d'études de la maladie et de pronostic de mort subite. La mutation du résidu arginine (Arg ou $R$ ) en position 403, l'une des plus fréquentes, est l'exemple le plus documenté de la variabilité des conséquences pathologiques des mutations ponctuelles dans les chaînes lourdes de myosine. En effet, une mutation du résidu arginine 403 a été repérée dans dix familles non apparentées. Elle donne naissance dans huit familles à une transformation en résidu glutamine ( $\mathrm{Gln}$ ou $Q)$. Dans la plupart de ces familles, le pronostic de mort subite est élevé alors que, dans un petit nombre de familles ayant la même mutation, elle a apparemment des conséquences moins dramatiques. Dans deux autres familles [16], l'arginine 403 est remplacée par un tryptophane ou par une leucine. Dans ce dernier cas, la mutation apparaît maligne et a des conséquences graves; dans le cas de la mutation en tryptophane, le pronostic semble beaucoup plus favorable (mutation bénigne). Il y a donc une grande variabilité de la pénétrance et de la gravité de la maladie en fonction du défaut génétique (nature et position de la mutation). Quant à la liaison causale entre ces mutations ponctuelles et l'hypertrophie cardiaque, celle-ci a été confirmée de manière quasi certaine par la mise en évidence de mutations de novo du gène de la chaîne lourde $\beta$ ventriculaire survenues, soit au niveau germinal, soit a u niveau somatique $\left(\mathrm{m} / \mathrm{s} n^{\circ} 1\right.$, vol. $\left.9, p .100\right)$.

au plan génétique de familles atteintes de cardiomyopathie hypertrophique $\left(\mathrm{m} / \mathrm{s} n^{\circ} 9\right.$, vol. $6, p$. 920) $([15,16]$; pour une revue et une bibliographie récente, voir [26]). Aujourd'hui, plus de vingt mutations ponctuelles ont été repérées le long de la chaîne lourde $\beta$ de myosine (voir figure 7A pour une représentation linéaire des mutations). Ces mutations impliquant les chaînes lourdes de myosine $\beta$ ventriculaire semblent responsables de la maladie chez $20 \%$ à $30 \%$ des malades atteints de CHF, et d'autres loci chromosomiques pour la $\mathrm{CHF}$ ont été identifiés: $1 \mathrm{q} 3,11 \mathrm{pl} 3$ et $15 q 2$. Les cardiomyopathies à myosine mutée présentent une grande hétérogénéité phénotypique. Les diverses mutations indiquées plus haut ont, en effet, des pénétrances variables à l'intérieur des familles atteintes et des conséquences variables en terme de gravité
La présence d'ARN messagers codant pour des chaînes lourdes de myosine $\beta$ mutée a été identifiée directement à partir d'extraits musculaires cardiaques ou grâce à l'amplification de transcrits ectopiques dans les lymphocytes de patients. Le messager mutant a également été mis en évidence dans le muscle squelettique lent de malades atteints de CHF. Tous ces résultats s'accordent en faveur de la coexpression des formes normales et mutées. Alors qu'au niveau du myocarde, une hypertrophie ventriculaire gauche ou septale d'amplitude variable est toujours détectée par échocardiographie, l'analyse microscopique des cardiocytes issus de régions hypertrophiées a révélé une organisation globalement intacte de la fibre cardiaque [27] et non pas une désorganisation 
myofibrillaire massive comme cela avait été préalablement observé. Au niveau de la protéine, des études de protéolyse limitée à l'arginase C laissent penser que la protéine mutée en 403 (R403Q) est exprimée dans le myocarde malade. La mise en évidence directe de la protéine mutée et l'étude morphologique directe de celle-ci dans le filament épais attendent toutefois que des anticorps spécifiques de la mutation soient disponibles.

La distribution des diverses mutations sur la structure cristalline de la myosine montre que les mutations sont réparties tout le long de la chaîne lourde et que l'on peut les regrouper autour de zones "fonctionnelles" (figure 7B). Certaines mutations affectent des résidus occupant des positions supposées fonctionnellement importantes. Par exemple, les résidus 403 et 249 , qui sont l'objet de fréquentes mutations aux conséquences morbides, se situent respectivement au bord de la gorge principale d'interaction avec l'actine et en plein milieu du site de fixation du nucléotide. L'étude de structures cristallines renfermant les diverses mutations connues, mais aussi d'autres mutations non détectées chez les malades à cause de leur effet létal, devrait permettre de préciser le rôle de chacune de ces mutations. Quelques études donnent actuellement des informations sur les conséquences fonctionnelles éventuelles de certaines mutations. Par exemple, il a été montré que la stabilité protéique de sept myosines mutées différentes n'est pas affectée dans des cellules non musculaires de mammifères (COS) transfectées par des constructions codant pour ces mutants [28]. Leur capacité de former des filaments semble cependant altérée: un tiers des cellules transfectées sont incapables de former des structures filamenteuses. Des différences significatives de motilité ont été obtenues entre des préparations de myosine normale et mutée. Les vitesses de glissement de l'actine sur les myosines totales issues de biopsies de muscle squelettique lent de patients, étaient réduites de trois à quatre fois. Toutefois, comme myosines normales et mutées sont toutes deux présentes dans l'extrait, l'effet

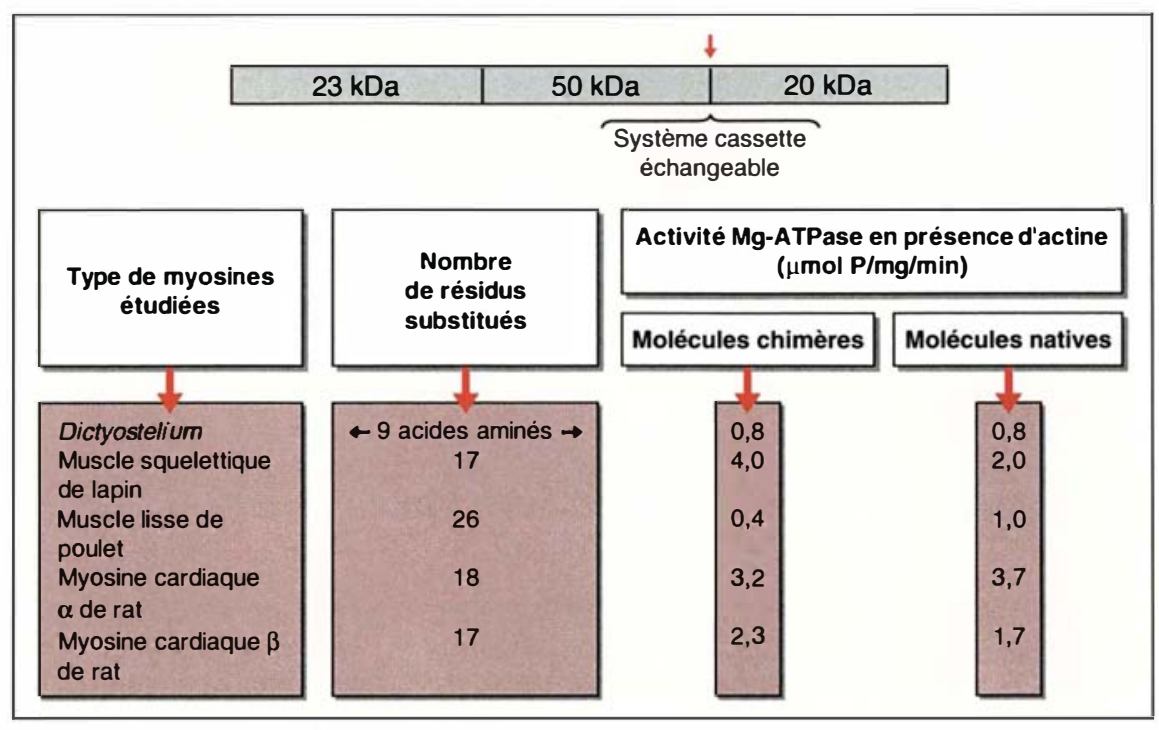

Figure 6. Construction par génie génétique de myosines ayant un court fragment chimérique variable à la jonction des fragments 50 et $20 \mathrm{kDa}$. La molécule de myosine est celle de Dictyostelium (myosine non musculaire). Le gène codant pour un fragment de chaîne lourde, issu de différentes myosines, est inséré à la place des 9 acides aminés présents dans la chaîne lourde de Dictyostelium. La zone d'insertion de la "cassette" se situe entre les résidus 613 et 621 de la chaîne lourde de myosine de Dictyostelium [14]. Les effets du fragment chimérique sur les activités ATPasiques dépendantes du magnésium en présence d'actine par rapport à celles obtenues pour les myosines natives correspondantes ont été notés. II apparaît que la présence de ce fragment de chaînes lourdes dans la myosine non musculaire transformée est déterminant pour la valeur de l'activité enzymatique mesurée.

blablement beaucoup plus important qu'on ne l'observe. Utilisant des quantités très faibles de fragments recombinants de myosine mutée (R403Q) exprimés dans des cellules d'insecte, Sweeney et al. [29] ont montré que ces mutants fonctionnaient de façon anormale [diminutions significatives de l'activité ATPasique activée par l'actine $(1 / 4)$ et de la vitesse de translocation des filaments d'actine $(1 / 5)]$. Ce résultat indiquant que la mutation altérerait directement le mécanisme d'interaction entre l'actine et la myosine n'a pas été retrouvé sur des fragments recombinants de la tête de myosine $\beta$ produits par E. coli [19]. Les reconnaissances élémentaires du nucléotide et de l'actine normalement obtenues avec ces fragments de chaîne lourde ne sont absolument pas modifiées par la mutation R403Q. Cela suggère que la mutation en 403 modifie le couplage (la transmission) entre les deux parties de la myosine situées de part et d'autre de la mutation, sans affecter les constantes de fixation du nucléotide et de l'actine.

\section{Perspectives}

Les observations qui viennent d'être décrites donnent donc une nouvelle jeunesse à la myosine! Les progrès réalisés sont dus à l'essor des méthodes de préparation et d'analyse des molécules, en particulier dans les domaines de la cristallographie, de la mécanique moléculaire, du génie génétique, de la cinétique enzymatique et de la génétique en physiopathologie. Alors que l'on croyait tout connaître de la myosine, plus d'un siècle après sa découverte, le développement de nouvelles méthodes d'analyse et les études menées en parallèle sur d'autres molécules sœurs, comme les kinésines et les dynéines, permettent de commencer à comprendre comment fonctionnent ces " moteurs biolo- 

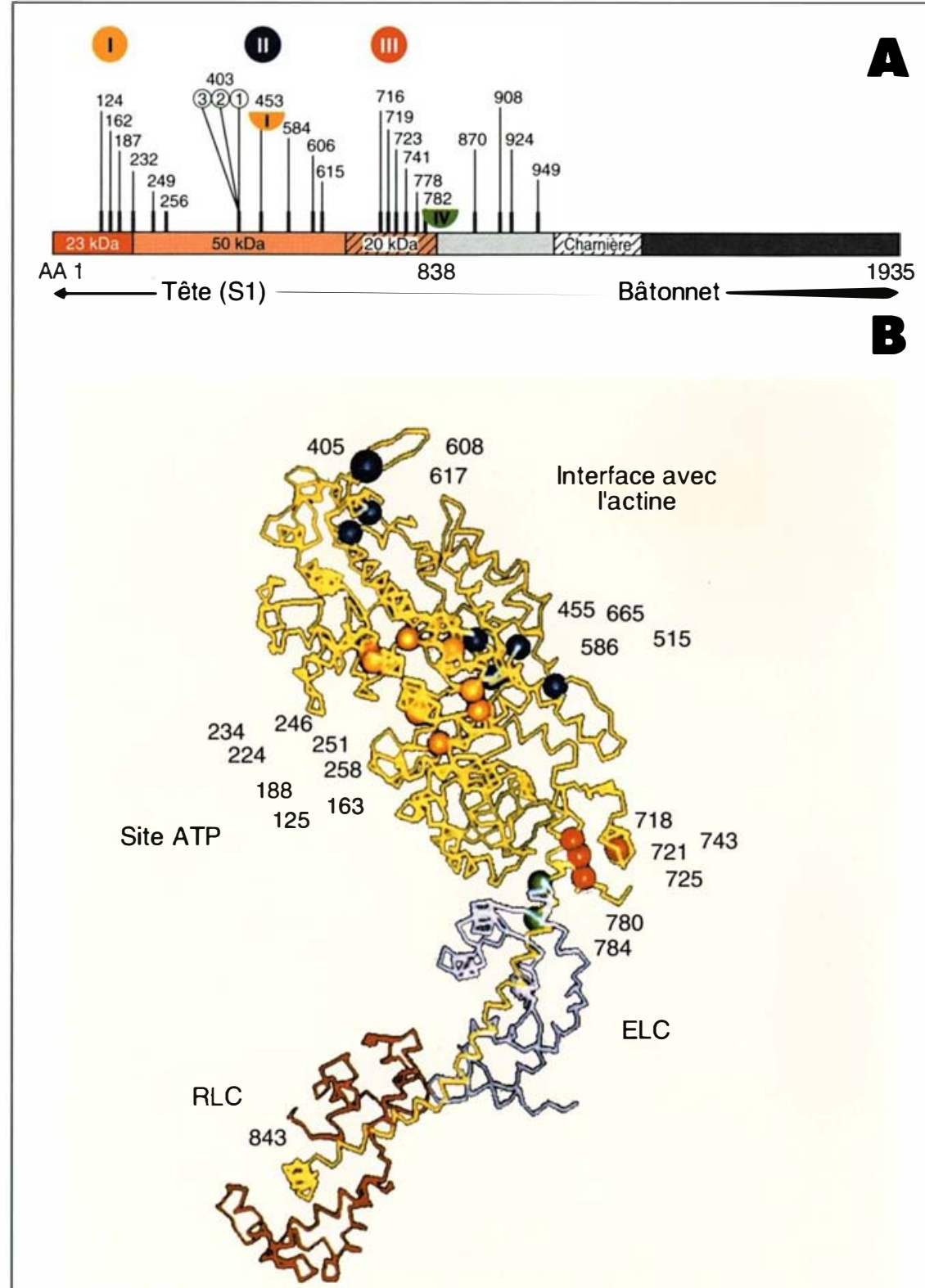

Figure 7. Mutations dans la chaîne lourde $\beta$ de myosine ventriculaire chez des patients atteints de cardiomyopathie hypertrophique familiale $[15,16]$. A. Répartition des diverses mutations ponctuelles le long de la chaîne lourde de myosine en représentation séquentielle. La numérotation des résidus est celle de la chaîne lourde $\beta$ majoritairement exprimée dans le ventricule humain. On a noté que trois mutations différentes ont été détectées pour le résidu 403. B. Répartition des diverses mutations ponctuelles sur la structure tridimensionnelle (carbones $\alpha$ uniquement) de la tête de la myosine squelettique de poulet $[5,6]$. La numérotation des résidus est celle de la séquence de la chaîne lourde de myosine de muscle squelettique rapide de poulet (résidus 1 à 843) (données issues de la Protein Data Bank, Brookhaven, NY, USA). La représentation a été réalisée avec le programme Insight II (Biosym, Technology Inc, San Diego, USA) par A. Aumelas (CBS, Montpellier, France). Les numérotations des mutations ont été placées en positions similaires à celles occupées dans la structure. Des décalages variables de quelques unités existent entre les deux séquences en comparaison. II faut noter que les mutations se répartissent en ligne to'st le long de la chaîne lourde de myosine et que l'on peut les regrouper autour de trois "zones" fonctionnelles principales dans la tête de la myosine indiquées par des couleurs différentes. giques" au niveau moléculaire. Le chemin à parcourir est encore long! Il faut encore rechercher les raisons de la diversité moléculaire des chaînes de myosine et prendre en compte sans doute davantage le rôle des autres partenaires impliqués dans l'acte contractile. L' “activité propre” du filament d'actine ainsi que les influences des nombreuses variantes observées dans la régulation de la contraction (par le filament fin, par les chaînes légères régulatrices...) sur les "fonctions" des diverses myo$m / s n^{\circ} 7$, vol. 1l, juillet 95 sines sont certainement toujours mal comprises. La combinaison du génie génétique et de la biochimie structurale est plus que jamais nécessaire pour comprendre les modes de communication entre ces molécules oligomériques complexes que sont les myosines et leurs nombreux partenaires. Ces approches fondamentales aideront également à décrire l'impact fonctionnel des mutations. La mise au point de lignées cellulaires ou d'animaux génétiquement modifiés en vue d'exprimer les muta- tions est également à mettre au programme. De tels animaux seront une source "incomparable" de molécules chimères permettant des études structure-fonction, impossibles à partir de tissus humains. Les animaux (transgéniques sans doute) pourraient être des modèles d'hypertrophie cardiaque héréditaire. On voit l'importance en santé publique de tels modèles permettant l'étude de l'étiologie de l'hypertrophie et de l'insuffisance cardiaque

Voir Summary p. 1016 


\section{Summary}

A new youth for myosins

The first two crystalline structures of the myosin head were recently published. The myosin head is made of two main domains. The motor domain comprises the first 600 N-terminal residues of the myosin head heavy chain; it contains the ATP-binding site and the main interface with actin. The transmission domain is composed of the long helicoidal C-terminal part of the myosin head heavy chain, along with essential and regulatory light chains. New motility assays demonstrated the movement and force produced by a single myosin molecule along the actin filament. Genetics and protein engineering led to the production of chimeric myosin molecules. These combined techniques enabled to demonstrate that the motor domain of the myosin head in skeletal muscles is self-sufficient for hydrolyzing ATP and inducing movement, and that the presence of light chains is crucial for motility efficiency by stabilizing the transmission domain. In contrast, light chains in non-striated muscle or non-muscle myosins determine motor activity by regulating ATP hydrolysis but do not markedly influence motile performances of the corresponding muscles. Such studies on the myosin structure and structure-function relationships between heavy and light myosin subunits helped to redefine the specific role of certain myosin domains. All these advances have generated renewed interest in the field of biological motor molecules and myosin isoforms. The discovery of point mutations in the ventricular myosin heavy chains from patients with familial hypertrophic cardiomyopathy has accelerated new research projects in this promising field!

\section{TIRÉS À PART}

